\title{
Reply to: A novel classification of aberrant right hepatic ducts ensures a critical view of safety in laparoscopic cholecystectomy by Kurahashi et al.
}

\author{
Luke Umana ${ }^{1}$ Eugene P. Ceppa ${ }^{1}$ (I)
}

Received: 26 April 2020 / Accepted: 29 April 2020 / Published online: 6 May 2020

(c) Springer Science+Business Media, LLC, part of Springer Nature 2020

Laparoscopic cholecystectomy is one of the most commonly performed procedures internationally. The benefits and pitfalls of the procedure are shared amongst patients and surgeons regardless of practice site or patterns. The gravest risk of any cholecystectomy is an iatrogenic bile duct injury. Since the advent of the laparoscopic cholecystectomy in the late $1980 \mathrm{~s}$, the reported incidence of bile duct injury is $0.3 \%(0.1-0.7 \%)$ and $80 \%(71-91 \%)$ of these injuries from misinterpretation of biliary anatomy $[1,2]$. This number has not improved over the last three decades despite surgical advancement in terms of treatment of injuries as well as enhanced training both in residency and advanced fellowships, surgical technology, and surgical awareness by individual surgeons and surgical societies such as SAGES with the Safe Cholecystectomy Task Force to avoid such injuries [3]. This paramount issue highlights the value of the manuscript titled "A novel classification of aberrant right hepatic ducts ensures a critical view of safety in laparoscopic cholecystectomy?" submitted to surgical endoscopy by Drs. Kurashi, Komatsu, Matsumura, Fukami, Arikawa, Saito, Osawa, Uchino, Kato, Suzuki, Toda, Kaneki, and Sano from the Department of Gastroenterological Surgery, Aichi Medical University, Nagakute, Aichi, Japan.

The authors performed a retrospective review of patients undergoing elective laparoscopic cholecystectomy at a single-institution over a 4-year period of time in which preoperative review of diagnostic imaging was used to identify the anatomy of the right hepatic duct. High-risk variations

This comment refers to the article available online at https://doi. org/10.1007/s00464-020-07610-8.

Eugene P. Ceppa

eceppa@iu.edu

1 Department of Surgery, Indiana University School of Medicine, 545 Barnhill Dr., Emerson Hall 541, Indianapolis, IN 46202, USA of the right hepatic duct were defined as Type A (communicating with the cystic duct) and Type B (traveling along the neck or $<5 \mathrm{~mm}$ from the infundibulum). Preoperative MRCP was reviewed to define the right hepatic duct anatomy based on Hisatsugu's classification [4]. The authors created their personal definition of high-risk to include any of the Hisatsugu's classifications in which the right hepatic duct was close to the dissection site during laparoscopic cholecystectomy. They converted Hisatsugu I $+\mathrm{V}$ to be Type A as defined as right hepatic duct communicating to the cystic duct, while Type B defined as and right hepatic duct along the neck or infundibulum of the gallbladder regardless of biliary tree confluence pattern. Any other case was described as low risk. Fifty patients (7\%) were found on preoperative MRCP or ERCP to have a variation of the right hepatic duct. Of these 50 patients, 4 were Type A and 12 were Type B deemed "high" risk, thus the other 34 were deemed "low" risk variations of the right hepatic duct. There were no demographic nor hepatic segment 5-7 drainage pattern differences between the two groups. The high-risk group had significantly more subtotal cholecystectomies performed than the low-risk group.

What is notable from this manuscript is that the authors were able to codify a particularly complex, yet pragmatic issue that all surgeons face when performing difficult cholecystectomies due to either arterial or biliary variation as well as intermittently associated with severe acute or chronic cholecystitis. As there is no dominant hepatic arterial pattern, variant hepatic anatomy should be expected and therefore the surgeon should have a high suspicion when critical structures are not easily identified during the critical view of safety dissection. Variant biliary anatomy has been shown to be an independent predictor of bile duct injury [5]. In a similar cohort size, $3 \%$ of patients with variant biliary anatomy had bile duct injuries while $0.3 \%$ of those with expected biliary anatomy had bile duct injuries with an OR of 16.56. The low incidence of bile duct injury suggested 
that subtotal cholecystectomy for this high-risk group could be deemed a safer approach and should not be just reserved for patients with severe cholecystitis. This study demonstrated a meticulous approach to discerning this anatomy with preoperative MRI as well as careful dissection due to heightened awareness.

The use of routine preoperative MRI/MRCP is one area that needs further investigation prior to implementation. At our institution, MRI/MRCP can cost approximately \$2500 USD. This price is particularly high for all patients being evaluated for a cholecystectomy especially in the setting of routine symptomatic cholelithiasis. In addition to MRCP, preoperative ERCP is a viable option to delineate variant anatomy because ERCP has been shown to be superior to MRCP in terms of cost effectiveness, albeit in the management of suspected biliary obstruction [6]. One option would be routine preoperative ERCP but this would have unnecessary increased exposure to risks such as additional general anesthetic, ampullary hemorrhage, and post ERCP pancreatitis as well as similar increased costs. Surgery-first treatment strategies, such as laparoscopic cholecystectomy with intraoperative cholangiography, are more cost-effective when compared to preoperative MRCP and ERCP in patients with symptomatic cholelithiasis with low probability of choledocholithiasis [7]. In general, any type of routine preoperative imaging other than transabdominal ultrasound is difficult to justify because of unnecessary cost and/or risk incurred to the patient.

The arching value of this study would be that the presence of variant high-risk right hepatic duct in suboptimal tissue planes then subtotal cholecystectomy should be seriously considered to avoid the morbidity associated with iatrogenic bile duct injury. Subtotal cholecystectomy is considered a safe option in patients with severe cholecystitis and when the components of the critical view of safety are not able to be achieved; this includes the known expected increased rates of biliary fistula and retained gallstones with subtotal cholecystectomy [8]. Future direction for this work would include a better way to select patients who would benefit from preoperative MRCP to determine high-risk anatomy or a series of steps intraoperatively to elucidate this anatomy without preoperative MRCP possibly via indocyanine green fluorescent cholangiography. We envision this as a natural adjunct and possibly the "fourth" component to the critical view of safety.

Funding This research did not receive any specific grant from funding agencies in the public, commercial, or not-for-profit sectors.

\section{Compliance with ethical standards}

Disclosures Luke Umana and Eugene P. Ceppa declare that they have no conflicts of interest or financial ties to disclose.

\section{References}

1. Pesce A, Palmucci S, Greca G, La Puleo S (2019) Iatrogenic bile duct injury: impact and management challenges. Clin Exp Gastroenterol 12:121-128

2. Pesce A, Portale TR, Minutolo V, Scilletta R, Li Destri G, Puleo S (2012) Bile duct injury during laparoscopic cholecystectomy without intraoperative cholangiography: a retrospective study on 1,100 selected patients. Dig Surg 29(4):310-314

3. Pitt HA, Sherman S, Johnson MS, Hollenbeck AN, Lee J, Daum MR, Lillemoe KD, Lehman GA (2013) Improved outcomes of bile duct injuries in the 21st century. Ann Surg 258(3):490-499

4. Hisatsugu T, Yamamoto H, Igimi H, Konomi K, Hurusawa T, Jimi M (1974) Anomaly of bile duct including aberrant right hepatic duct with cholecystolithiasis. J Adult Dis 4:581-586

5. Natsume S, Kato T, Hiramatsu K, Shibata Y, Yoshihara M, Aoba T, Aiba T, Takada A (2017) Presence of aberrant anatomy is an independent predictor of bile duct injury during cholecystectomy. Int Surg 102(5-6):250-257

6. Adam V, Bhat M, Martel M, da Silveira E, Reinhold C, Valois E, Barkun JS, Barkin AN (2015) Comparison costs of ERCP and MRCP in patients with suspected biliary obstruction based on a randomized trial. Value Health 18(6)767-773

7. Epelboym I, Winner M, Allendorf JD (2013) MRCP is not a costeffective strategy in the management of silent common bile duct stones. J Gastrointest Surg 17:863-871

8. Elshaer M, Gravante G, Thomas K, Sorge R, Al-Hamali S, Ebdewi H (2015) Subtotal cholecystectomy for "difficult gallbladders": systematic review and meta-analysis. JAMA Surg 150(2):159-168

Publisher's Note Springer Nature remains neutral with regard to jurisdictional claims in published maps and institutional affiliations. 